\title{
VIRTUALMENTE INTERCULTURALES. LA EDUCACIÓN SUPERIOR EN BOLIVIA
}

\section{Introducción}

En la altiplanicie boliviana, otrora escenario de la civilización Tiwanacota, se halla un centro ceremonial que, en su entrada, tiene una plazoleta abierta y semisubterránea. En sus paredes internas se encuentran piedras que parecen cabezas clavas talladas y que la ciencia no ha podido explicar hasta ahora. ¿Cuál el misterio? Sus rasgos son tan variados que, a decir de algunos, podrían ser representaciones de los pueblos conquistados, y, a decir de otros, la representación de las etnias del planeta: desde un hombre con turbante, pasando por uno con ojos rasgados, hasta otro de color más oscuro y cabello rizado. Lo que no es misterio es que están allí, miles de años, proponiendo que en el planeta somos diversos y que, alrededor de una plaza o en las pantallas modernas de televisión, el ser humano convive con sus símiles.

Probablemente ese el antecedente más lejano para hablar de interculturalidad y educación superior en Bolivia, un país que aparece con más frecuencia en los medios masivos de información por la reciente asunción al mando presidencial de un mestizo con rostro autóctono, alejado, por lo menos en su proyección internacional, de los parámetros clásicos del "blanco" y con una forma de actuar que no encaja necesariamente en los estereotipos del diplomático francés del siglo XVIII ni en el manejo del Estado modernista y capitalista tradicional.

Lo curioso, por otro lado, es que Bolivia, luego de los violentos hechos políticos de revuelta popular que terminaron en octubre de 2003 con la dramática huida del ex presidente Gonzalo Sánchez de Lozada hacia Estados Unidos, ha comenzado a mirar también con ojos y conciencia hacia adentro. Es decir, ha visto que su cotidiano 
era per se diverso y conviviente con "esos otros" y, aunque todavía hay quienes se resisten, el cambio se está dando en todos los niveles de la vida y, por ende, de la cultura.

Uno de esos ámbitos es la educación superior, quehacer de los bolivianos como de tantos otros pueblos. Con una tradición universitaria señorial, iniciada en 1624 con la fundación de la primera universidad de la Audiencia de Charcas, territorio de los virreinatos de La Plata y de Lima, bajo el título nobiliario de Pontificia Universidad Mayor de San Francisco Xavier, y con una historia acompañada de sus universitarios en los momentos álgidos de las luchas por el poder.

Con 182 años de vida independiente, Bolivia se reconfigura nuevamente, impulsando una Asamblea Constituyente en la que el tema educativo no está ausente y menos el de la interculturalidad. Es más, las actuales condiciones del país están provocando que "lo intercultural" sea una transversal en todos los planes y en todas las suspicaces miradas.

Mas, ¿cómo entender lo intercultural (y su sistema educativo) en un país por tradición diverso pero, a la vez, por historia, llevado a borrar sus diferencias para homogeneizar ideologías y sus consecuentes ejercicios de poder? ¿Cómo entender el conocimiento y respeto del otro o de los otros en un país en el que la corriente lleva las aguas hacia la intolerancia, mediante sistemas de exclusión social y económica de sus pobladores, primero originarios, luego mestizos, a través de sus mecanismos de educación formal? ¿Cómo construir un sistema educativo, menos aún uno de educación superior, si por tradición impuesta bajo el euroconcepto de la conquista, las expresiones culturales y los saberes de los 36 pueblos indígenas reconocidos legalmente no responden a los cánones del academicismo positivista y enciclopédico que se inculca en el aula científica? Por último, ¿cómo pretender la imposición de las sabidurías occidentales y globalizadas bajo el nombre de "ciencia" frente a conocimientos ancestrales regidos por rituales y creencias con título de misterio e indigenismo que todavía se ejercitan en la vereda opuesta a la academia? 
Como las posibles respuestas dan para largo, propongo varios ámbitos atractivos para analizar - de modo somero- el tema central que esta revista propone acerca de la relación de lo intercultural con la educación superior y esto es plantearse -para el caso de Bolivia- más bien hechos que nos den razón o por lo menos justificación: la convivencia de culturas (a pesar de todo), las lenguas predominantes, los conocimientos compartidos, las experiencias logradas y los resultados bajo observación que están construyendo el ser intercultural boliviano.

Aclaro de inicio, para no crear falsas expectativas, que todo en Bolivia sigue en construcción y que la forma cotidiana de relaciones está regida por el sentimiento de que "hay mucho por hacer", sistema que permite la interacción de formas culturales diversas que no dependen sólo de la percepción de lo indio sino de "otras formas culturales" creadas que conviven a partir de ese mecanismo de exclusión que la "cultura oficial" ha generado.

\section{Brillo general con espejos regalados}

La educación superior en Bolivia se mantuvo, hasta la década de los años 80 exclusivamente a cargo del Estado nacional, bajo un régimen de autonomías administrativas y académicas implementadas desde la década de los 30, cuyos recursos se canalizan, todavía, a través del Tesoro General. La única excepción legalmente permitida hasta esa época fue la Universidad Católica Boliviana, de propiedad de la Iglesia Católica y con autonomía administrativa, académica y de manejo de recursos.

Sin embargo, la apertura democrática desde 1982 y los cambios continuos en el modelo socioeconómico del país permitieron la consolidación de un sistema de educación superior privado, solventado desde la misma empresa privada, como la Universidad Privada de Santa Cruz, o desde la visión evangelizadora, como la Universidad Evangélica Boliviana, ambas ubicadas en Santa Cruz de la Sierra, territorio próspero económicamente pero carente de recursos 
humanos propios que respondieran, además, al modelo de vida que entraba en vigencia.

En la ciudad de La Paz, sede del gobierno, las iniciativas privadas respondieron a la necesidad de formación especializada, en cortos periodos y exentos de la burocracia de una universidad estatal. Cochabamba, tercera ciudad en importancia económica del país en ese momento, implementó centros de formación con ofertas de carreras rápidas y una parafernalia de mercadeo.

Para la década de los 90, la educación terciaria se convirtió en un negocio y fueron pocas las universidades que optaron por una propuesta seria, acorde con las necesidades del país. Ante la eminente corrupción del sistema socioeconómico, algunas universidades ganaron prestigio por su solvencia, sus programas, su actualidad en docencia y demostrada seriedad académica. Otras aún debaten su existencia. Para finales del siglo XX, la educación superior había cambiado también su visión de servicio lucrativo y pasó a ser, en general, una actividad con mayor exigencia, porque la sociedad comenzó a demandar calidad, especializaciones, tecnología y una formación más cercana a las propias culturas.

Como seguramente sucedió también en otros países, en Bolivia se impuso, hasta muy entrado el siglo XX, un sistema de educación superior basado en los conocimientos y parámetros europeos del enciclopedismo. Pero este nuevo siglo trajo un mirar universitario hacia lo ancestral, rescatando desde tradiciones y formas de expresión artísticas hasta conocimientos y sabidurías que pusieron en contradicción al sistema educativo en general.

Esto lleva a la principal reflexión de este trabajo: en un país multicultural, que convive con un sistema educativo oficial monocultural, es necesario repensar "lo intercultural" desde otras perspectivas. Por ejemplo, en el área de la formación en salud las culturas originarias han pasado sus conocimientos, de generación en generación, a través de kallawayas o médicos naturistas. Charazani, una 
población limítrofe entre la puna y la zona subtropical, se constituye en el centro de formación no oficial de los varones kallawayas. Su proceder es relacionado por la cultura occidental con la magia y el ritual, y no fue ampliamente divulgado sino hasta la aparición de expresiones de reivindicación que se dieron entre 1975 y 1980, época en la que se instaló un consultorio de atención de medicina natural en el centro urbano de la ciudad de La Paz. Sin embargo, recién en este siglo se ha permitido a este centro la posibilidad de formar médicos naturistas con el nivel académico de técnicos, lo que ya es un avance en la idea de respetar y valorar a las culturas, aunque el pensamiento eurocentrista aún debate los contenidos académicos.

Como en el caso anterior, la educación superior para el área de las artes y la arquitectura ha rescatado también la necesidad de integrar el quehacer científico con la sabiduría tradicional, sobre todo indígena, a través de proyectos específicos de rescate de materiales de construcción, estilos de construcción, diseño de espacios y otros conocimientos que son incorporados en los planes de estudio de los arquitectos. El área de las ciencias jurídicas debate hoy en día el valor de la llamada "justicia comunitaria" y su interrelación con las normativas constitucionales copiadas de las Cartas Magnas francesas. En las otras profesiones, la formación en educación superior recién comienza a incorporar el término "intercultural" en sus planes de estudio, como reflejo de una realidad a todas luces cambiante o como un gancho académico para lograr clientes.

El caso de las carreras de comunicación en las universidades de Santa Cruz de la Sierra es muy interesante este año 2007, porque varias de ellas son de propiedad privada y han incorporado en su oferta académica la materia de Comunicación Intercultural, cuyos contenidos están relacionados tanto con los procesos de interculturalidad como tales, como con los procesos de comunicación que responden a la realidad boliviana: una convivencia pluri/multi de culturas.

Sin embargo, en las universidades en general no existe una interrelación cultural que permita todavía hablar de procesos 
interculturales como tales sino, simplemente, de reflexiones que terminan siendo, desde todas las áreas, sólo espejos que brillan y dejan ver partes de la realidad reflejados en ellos; espacios que, intuitivamente, están abriendo el escenario para generar interculturalidad.

\section{Atisbos y apropiaciones de lo intercultural}

La reflexión sobre la interculturalidad en el sistema de educación superior resulta ambivalente, entonces, en el caso de Bolivia: por un lado, la interculturalidad como proceso natural que convive en la calle y el cotidiano, y que se da en el saber popular influyendo sobre el saber científico (la hoja de coca que es prescrita en su forma de infusión por el médico, la orientación del techado o de las ventanas en una casa de acuerdo con la sabiduría popular, muy en contra de la teoría de resistencia de materiales, por ejemplo), y, por el otro, como procesamiento académico, de rigurosa definición, que requiere de institucionalización para ser aceptado por la academia no intercultural.

A estas alturas, resulta difícil hablar, entonces, de procesos de interculturalidad en la educación superior cuando la realidad es, de por sí, multi y pluricultural, producto de la existencia de 36 naciones indígenas. El 43\% de la población no reside en el lugar donde nació, influencia de: migraciones internas del área rural hacia las urbes hasta en $75 \%$, en relación con datos de hace diez años, que generan intercambio de conocimientos y aprendizajes diversos; migraciones del área occidental del país (puna, altiplano y valle), hacia las zonas subtropicales y tropicales (amazónicas) del oriente de Bolivia; presencia de grupos de blancos, bronceados, negros e indios en las festividades populares de carnaval o en las hibridaciones religiosas de "Todos los Santos".

Por ello, introduzco aquí un límite de rigurosidad operacional (aunque no por eso una adhesión de hecho): la interculturalidad se entiende como la convivencia y la interacción respetuosa y tolerante de expresiones culturales, a partir de condiciones de equidad, de justicia y de participación. 
Desde esta definición, en Bolivia se darían procesos de interculturalidad con altos grados de dificultad. Porque no hay equidad -por ejemplo- al verificar la presencia de indios, mestizos y blancos en las universidades o en los directorios de instituciones de poder económico como la Confederación de Empresarios Privados de Bolivia o la Cámara Agropecuaria del Oriente; no se podría dar la interculturalidad porque no se hace justicia con los que tienen menos ingresos económicos; hasta el término justicia está bajo observación. No podría haber interculturalidad porque, de acuerdo con el último estudio sobre diversidad cultural (UNIR, 2006), el color de la piel, pertenecer a un pueblo indígena y el apellido siguen siendo considerados los principales factores de discriminación en Bolivia. Esto lleva a altos niveles de intolerancia y negaciones de la existencia del "otro" y, con ese concepto, siempre estaremos pensando en convivencias culturales tensas y discriminadoras. Plantear, por tanto, la interculturalidad desde esa sola perspectiva teórica es arribar a la utopía y no dejar que las realidades hablen.

Desde otra óptica, menos rigurosa, la interculturalidad podría darse en términos simples de relaciones humanas cotidianas, en el sentido de compartir saberes, apropiarse de usos, adecuar o "acondicionar" los conocimientos y aplicarlos en la sobrevivencia. Estas relaciones, vistas las limitaciones de la universidad en general, parecen más bien reproducirse en centros de formación técnica superior, en los cuales comparten quienes quieren una profesión rápida, un oficio o, simplemente, un pasatiempo.

En los últimos años se ha incrementado la instalación de centros de estudio superior técnico como una respuesta al requerimiento de obtener un conocimiento rápido que permita un oficio, generalmente de tipo especializado, como las escuelas de mecánica, carpintería o computación, entre otras. Estos centros intercambian experiencias desde la sabiduría del migrante indígena, pasando por la experiencia del mestizo y terminando en la teoría del blanco; todos aglomerados alrededor de un motor, de una sierra sinfín o de una jeringa, como es el caso de las unidades académicas campesinas que, entre sus 
carreras, tienen enfermería y agropecuaria. Se convierten en agentes culturales que interactúan bajo condiciones similares de acceso a la información, participación y búsqueda de nuevos conocimientos. Allí hay atisbos de interculturalidad más real. Si de allí nacen los respetos, las tolerancias y las justicias, entonces podría ser que la educación superior no universitaria esté congregando mayores escenarios de interculturalidad.

En resumen, hay atisbos de interculturalidad en la educación superior formal, a través de la inclusión de pensamiento propio, generado a partir del rescate de saberes de otras culturas. Hay conceptos propios sobre la interculturalidad que se están anexando cada vez con más fuerza a los programas y contenidos de profesionalización, pero todavía no hay una aceptación tácita y general de la presencia e integración de lo intercultural en las formas de pensamiento de la comunidad científica boliviana.

Por otro lado, existen integraciones de lo cotidiano en la educación superior no universitaria a partir de las convivencias y de la elaboración de programas de formación en niveles técnicos, como en los casos de las unidades académicas campesinas de la Universidad Católica Boliviana, o a través de los centros privados y estatales de formación técnica, como el Instituto de Formación y Capacitación en Mano de Obra (Infocal), las escuelas de capacitación automotriz, la Escuela Hotelera y otras.

\section{Experiencias con ribete académico}

Dada la dificultad teórica de comprender lo intercultural en un país como Bolivia, o, mejor, dada la realidad empírica que revela otras dimensiones de lo intercultural y, de allí, la dificultad para configurar un estudio sobre el tema en la educación superior, es pertinente revisar las experiencias que tienen esos brillos.

Por un lado, los 20 años de las unidades académicas campesinas de la Universidad Católica Boliviana y de las que se crean bajo su techo 
académico, pero son de funcionamiento administrativo y financiero autónomos; por otro, los programas de educación intercultural bilingüe que tienen sus raíces en los cursos de idiomas nativos implementados hace 25 años por la Universidad Mayor de San Andrés (La Paz), y que han sido amplificados por la Universidad Mayor de San Simón (Cochabamba), a través de un programa de Formación de Educación Intercultural Bilingüe (PEIB); y, recientemente, la experiencia de investigación titulada "Entre la comunidad y la universidad"1, que escudriñó la construcción de identidad en la asimilación y articulación de valores, creencias y actitudes de los universitarios becarios de la Federación Sindical Única de Trabajadores Campesinos de Cochabamba, que estudiaban en la Universidad Mayor de San Simón y que ha dado luces para repensar la relación de lo indígena y sus formas de construcción del conocimiento comunitario y su actuar como formas de resistencias e integración al mundo occidental.

\section{Un vistazo al Programa de Educación de Interculturalidad Bilingüe (PEIB)}

A pesar de que el PEIB no es en sí una experiencia de educación superior, sino que nace en ella para beneficio de la formación de profesores del ciclo básico y luego de niños y niñas en edad escolar, es oportuno apuntar algunos aspectos:

1) El programa tiene antecedentes en la actividad política de la Confederación Nacional de Maestros de Educación Rural de Bolivia (CONMERB), fundada en 1966, que en la década de los ochenta "reivindicó la necesidad de una educación que reconociese la conveniencia de recurrir a las lenguas y culturas indígenas y, lo que es más, el derecho de las poblaciones indígenas a una educación en lengua propia y desde su propia cultura" (López, 2000).

2) Según un informe de Luis López (2000), evaluando el PEIB, a partir de los 80 "los ideales de la interculturalidad y el bilingüismo,

1 Beca otorgada por el Programa de Investigación Estratégica en Bolivia (PIEB) al equipo de investigadores coordinado por Lourdes Saavedra en 2005. 
y la temática indígena, en general, se instalan en el discurso pedagógico y político bolivianos y comienza a avizorarse una manera distinta de mirar la relación indígenas-no indígenas, fundamentalmente a partir de la esfera educativa" (López, 2000: 14). Claras aplicaciones están en la organización del Servicio Nacional de Educación Popular "Elizardo Pérez" (SENALEP), que elaboró cartillas educativas en idiomas nativos, base, a su vez, de la Reforma Educativa de 1994.

3) El PEIB se crea posteriormente con apoyo de UNICEF-Bolivia. Trabaja en lo pedagógico bajo la óptica de mantenimiento y desarrollo de las lenguas originarias, y, en lo cultural, desde una perspectiva de interculturalidad.

4) Este programa ha influido en la reforma educativa actual, que tiene como sus dos ejes principales la interculturalidad y la participación popular, y en varios cambios en la Constitución Política del Estado.

5) Por último, otra influencia directa ha sido la creación de la Licenciatura en Educación Intercultural Bilingüe en la carrera de Ciencias de la Educación de la Universidad Mayor de San Simón, en Cochabamba, experiencia que ya tiene resultados atractivos, tanto por la orientación de lo intercultural a través de lo lingüístico como por la participación de indígenas en diferentes estamentos académicos, desde el alumnado hasta la planificación curricular.

\section{Unidades académicas campesinas}

Las unidades académicas campesinas (UAC) son fundadas en 1987 por iniciativa de la Universidad Católica Boliviana (UCB), en el marco de sus principios, filosofía y misión de formar profesionales capacitados y comprometidos con la sociedad boliviana.

Hasta 1994 se consolidan como un subsistema de educación superior en las regiones de Batallas, Pucarani y Tiahuanaco, que pertenecen al Altiplano Norte del departamento de La Paz, 
implementando primero carreras con el grado de técnico superior y, luego, licenciaturas en Agroindustria, Agropecuaria y Enfermería. Posteriormente, en 1994 y 1996 se crearon las UAC de Carmen Pampa, en la provincia Nor Yungas (cabecera de valle) y la de Escoma, en la provincia Camacho, con las carreras técnicas de Turismo Rural (en Carmen Pampa), Agropecuaria y Tallado en Madera y Ebanistería (en Escoma); además de las carreras de Ingeniería Agronómica, Enfermería, Medicina Veterinaria y Zootecnia y Educación (en Carmen Pampa), y Educación (en Escoma).

Las tres primeras UAC se hallaban bajo la administración y financiamiento de la UCB, y las dos restantes cuentan con el techo académico y la administración de instituciones sociales.

Su principio de funcionamiento fue la recuperación de conocimientos tradicionales y su integración con conocimientos académicos y técnicos del mundo moderno, sin entrar en conflicto con los valores de las comunidades donde se instalaron.

"El concepto de Unidad Académica Campesina se sustenta, fundamentalmente, en cubrir las necesidades del sector rural y del contexto social, a las que la universidad establece una respuesta mediante una formación académica con una profesionalización en áreas técnicas y otros niveles con los valores humanísticos universales, donde el proyecto en su programación de actividades toma muy en cuenta el sentido de la dinámica comunitaria de la zona, lo que facilita la participación de los miembros que componen las actividades académicas de las UAC" (Secretaría Académica, Unidad Académica Campesina, UCB, 2007).

Las UAC han sido reconocidas por Organización de las Naciones Unidas como un modelo de formación relacionado con el concepto de desarrollo humano sustentable para suprimir la pobreza, debido a los estándares académicos rigurosos que se enseñan a los estudiantes indígenas de cada región, según afirman en la UCB. 
El criterio de la formación académica está acompañado, además, de la lógica de "devolución del conocimiento a la comunidad". Es decir, a quienes asisten a las UAC se les inculca el concepto de trabajo para el desarrollo de su propia comunidad como una transversal en asignaturas, trabajos y actividades curriculares en general. Esto ha provocado una reflexión en otras experiencias acerca de la influencia de las UAC en la migración y en el retorno de migrantes a las zonas campesinas, elemento que también construye "lo intercultural" en la educación superior.

Desde este punto de vista, probablemente se pueda hablar de una integración cultural de los actores participantes en los procesos de enseñanza-aprendizaje, incorporando en cada acción "al otro". Sin embargo, queda pendiente la producción intelectual y científica que se haya generado como verdaderos procesos interculturales. En Carmen Pampa, para descargo, se elaboraron las dos primeras tesis en idioma aimara ${ }^{2}$, lo que ya le agrega un valor intercultural importante a los resultados académicos.

A fines del siglo pasado, una reflexión interna a la UCB acerca de los tiempos invertidos versus los resultados, las proyecciones y las inversiones concluyó que un grupo importante de alumnos (campesinos) no finalizaba su profesionalización porque priorizaba su trabajo agrícola o porque migraba a las ciudades más grandes. Como respuesta se implementaron -y con excelentes resultados-, los cursos de peritos de corta duración.

A partir de esas experiencias, las cifras hasta 2006 muestran que se inscribieron 1.996 alumnos, egresaron 786 y se titularon 460 en los cursos regulares de técnicos y licenciaturas, mientras que el total de peritos llega a 1.934, indicando una importante actividad que refleja integración cultural, aunque todavía deja bastante espacio para profundizar la reflexión sobre su dimensión intercultural.

2 Que no tiene realmente una construcción lingüística escrita sino una adaptación fonética a las grafías y estructuras usadas en el castellano. 


\section{Un destello en el horizonte cercano}

El Programa de Investigación Estratégica para Bolivia (PIEB) patrocinó en 2004 el trabajo "Entre la comunidad y la universidad: jóvenes campesinos universitarios, interculturalidad e identidad en la Universidad Mayor de San Simón" (Saavedra, 2005), que se ha constituido en hito en estos últimos años en el tema de educación superior e interculturalidad, tanto por los resultados como por la reflexión.

El problema de investigación que se planteó el equipo se definía de la siguiente manera: “¿Cómo los universitarios becarios de la Federación Sindical Única de Trabajadores Campesinos de Cochabamba construyen su identidad a partir de sus relaciones con instituciones, organizaciones sociales e individuos en el proceso de conformación de su organización de universitarios?" (Saavedra, 2005), propuesta que no indagó en los programas de educación superior sino en la vivencia misma de los indígenas mientras se formaban en la Universidad Mayor de San Simón, en Cochabamba.

Además de descubrir que la reflexión sobre interculturalidad en la educación superior boliviana no se había profundizado, sino que se habían seguido modelos que sólo permitieron la reproducción de conocimiento, estos investigadores aportaron una serie de datos para la comprensión del proceso de construcción identitaria como, por ejemplo, los espacios donde se verificaba interacción o exclusión cultural, la preeminencia del concepto sindical (probablemente un saber comunitario) en las relaciones jerárquicas y de organización de los "becarios indígenas", o los mecanismos ligados a las resistencias culturales.

En cuanto a la discriminación, el informe preliminar de los investigadores ya apuntaba:

"A pesar de que varios estudiantes ingresaron por méritos académicos a la universidad, esto no es una garantía para que se destaquen en las carreras que estudian, ya que, al proceder de colegios en su mayoría fiscales, no están lo suficientemente preparados para 
cubrir las exigencias de la universidad (...) La discriminación (por el momento) fue detectada en el sector de los docentes y auxiliares (...) En lo que respecta a las relaciones con sus compañeros (por el momento), los entrevistados señalan no haber experimentado discriminación de manera manifiesta, por lo general no hay una aceptación ideal hacia ellos, como lo señala una estudiante de comunicación: "siempre hay jailones, pero ellos están con su gente y no pasa nada, luego nos hablan también" (Saavedra, 2005).

La investigación también reveló que el ingreso de los jóvenes campesinos a la universidad no era el final de una conquista grupal, "sino el principio de una larga lucha por pertenecer a una institución de educación superior que no tiene la capacidad necesaria para gestionar programas, grupos de reflexión, cuerpos docentes que ayuden a sectores como los estudiantes de provincia" (Saavedra, 2005).

En el informe de referencia se lee: "un becario señaló que ellos estudian más y que los otros no hacen nada, que harán respetar sus derechos como cultura, "sabemos de donde venimos", deduciendo de esas frases que la presencia indígena en la universidad se transforma también en un importante elemento político de organización, movilización y crítica respecto al poder" (Saavedra, 2005).

A través de las entrevistas personales, los investigadores descubrieron que los campesinos cochabambinos eran hijos de migrantes de zonas mineras como Potosí y Oruro o de sectores agrícolas de La Paz, lo que evidenciaba un contexto de exclusión y discriminación, y, como ellos mismos confirmaron, de ámbitos situados entre "la comunidad y la universidad".

\section{El ser virtualmente intercultural}

Un resumen como el presentado acerca de la educación superior en Bolivia puede dejar sabor a virtualidades, escueto tratamiento que tiene, en resumen, dos polos: lo intercultural visto desde la formación profesional, en la que el indígena participa reproduciendo 
conocimientos en su comunidad, con atisbos de equidad mientras aprenda la ciencia, y lo intercultural visto desde la resistencia que pretende apropiarse del conocimiento existente para reformularlo en su interacción con el mundo. En otra dimensión, lo intercultural desde el bilingüismo se constituiría en elemento técnico que todavía proporciona mucha materia para reflexionar.

Mientras tanto, en castellano, quechua, aimara o guaraní, los bolivianos interactúan en los centros de educación superior en mil formas, intercambiando saberes y conocimientos, vidas imbricadas en respuesta a sus cotidianos de sobrevivencia. Desde migrantes que pasan rápidamente de ser campesinos a internautas, mediatizados por la clonación de un celular o la piratería de un CD, hasta destacados y reflexivos conferencistas que trabajan en procura de sistematizar su conocimiento ancestral para hacerlo compatible con la ciencia "oficial". Toda una amalgama de colores que da vida a seres virtualmente interculturales, como el país.

\section{Referencias bibliográficas}

Fundación UNIR Bolivia. (Junio 2006) Casa Común. Estudio Diversidad Cultural. La Paz, Bolivia.

López, Luis Enrique. La educación de jóvenes y adultos indígenas en Bolivia. Informe Final. Disponible en http://www.unesco.org/education/uie/ pdf/Bolivia.pdf Último acceso en mayo de 2007.

Saavedra, Lourdes et al. Entre la comunidad y la universidad: jóvenes campesinos universitarios. interculturalidad e identidad en la Universidad Mayor de San Simón. La Paz: Programa de Investigación Estratégica para Bolivia. PIEB. 2005. Informe de avance disponible en www.pieb.org/campe sinosenlauniversidad/contents/pre\%20INFORME\%20AVANCE.ppt Último acceso en mayo de 2007.

Secretaría Académica, Unidad Académica Campesina, UCB. (12 de marzo de 2007) Al servicio de la educación superior en el área rural. Documento enviado a hurtadoal@yahoo.es

Recibido: 17 abril de 2007

Aceptado: 4 mayo de 2007 